\title{
Nasopharngeal Tuberculosis Simulating Cancer: About 2 Cases
}

\author{
F. Hadid, O. Oulghoul, H. Benjelloun, O. Benhommad, Y. Rochdi, and A. Raji
}

\section{ABSTRACT}

Primitive tuberculosis of the nasopharynx is rare. Clinical symptoms and paraclinical examinations are often confusing. Pseudotumour forms are frequent, which poses a problem of differential diagnosis with cavum cancer. We reported two cases of nasopharyngeal tuberculosis, followed up in the ear, nose and throat department of the Arrazi Hospital of the Mohammed VI University Hospital in Marrakesh. The first case concerned a 22-year-old female patient admitted to hospital for an etiological assessment of a right spinal adenopathy evolving in a context of altered general condition with weight loss, anorexia, and night sweats. The second case was a 14-year-old patient with a fistulised right spinal adenopathy with nasal obstruction and homolateral deafness. Nasofibroscopy in both patients showed a burgeoning tumour on the right posterolateral wall. A biopsy of the cavum was indicated. The histological study of the biopsies revealed an epithelial-giganto-cellular granuloma with caseous necrosis. This allowed to make the diagnosis of nasopharyngeal tuberculosis, and an antibacillary treatment was instituted. The evolution was favorable.

Keywords: Tuberculosis, nasopharyngeal, nasofibrosopy, histology.
Submitted : May 02, 2021

Published : May 28, 2021

ISSN: 2593-8339

DOI: 10.24018 /ejmed.2021.3.3.852

\section{F. Hadid*}

Ear, Nose and Throat Department, Mohammed VI University Hospital,

Marrakesh, Morocco.

(e-mail: fz.hadid@ ${ }^{@}$ gmail.com)

O. Oulghoul

Ear, Nose and Throat Department, Mohammed VI University Hospital,

Marrakesh, Morocco.

H. Benjelloun

Ear, Nose and Throat Department,

Mohammed VI University Hospital,

Marrakesh, Morocco.

O. Benhommad

Ear, Nose and Throat Department, Mohammed VI University Hospital,

Marrakesh, Morocco.

Y. Rochdi

Ear, Nose and Throat Department, Mohammed VI University Hospital,

Marrakesh, Morocco.

A. Raji

Ear, Nose and Throat Department,

Mohammed VI University Hospital,

Marrakesh, Morocco.

*Corresponding Author

\section{INTRODUCTION}

Tuberculosis (TB) is an infectious disease caused by the bacillus mycobacterium tuberculosis, it typically affects the lungs (pulmonary TB), but can affect other sites as well (extra pulmonary tuberculosis) [1]. Nasopharyngeal tuberculosis is rare type of extrapulmonary tuberculosis comprising only less than $1 \%$ of tuberculosis found in the upper respiratory tract [2]. Tuberculosis often presents in the head and neck, with the cervical lymph nodes being one of the commonest sites of extrapulmonary tuberculosis. Head and neck tuberculosis also present in the oral cavity, nose, ears, larynx, thyroid, and salivary glands.

\section{Clinical CASES:}

\section{A. Case File 1}

Patient aged 22 years, without any particular pathological history, with the notion of tuberculosis contagion (2 sisters treated for pulmonary and lymph node tuberculosis). The patient consulted for a right spinal adenopathy, evolving in the last 3 months, progressively increasing in volume in a context of unquantified weight loss. The clinical examination showed a $2 \mathrm{~cm} / 2 \mathrm{~cm}$ right spinal adenopathy, mobile, painless, without inflammatory signs, with rhinocavoscopy (Fig. 1): a budding tumour on posterolateral wall of the cavum, which prompted us to perform multiple biopsies under local anaesthesia, and whose histological diagnosis showed the presence of an epithelio-gigantocellular granuloma with caseous necrosis (Fig. 2, 3), without associated signs of malignancy. The biological assessment showed an inflammatory syndrome.

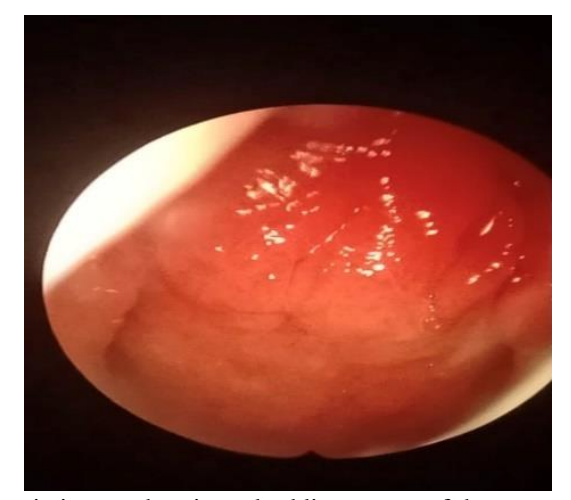

Fig.1. Endoscopic image showing a budding tumor of the post-cavum wall. 


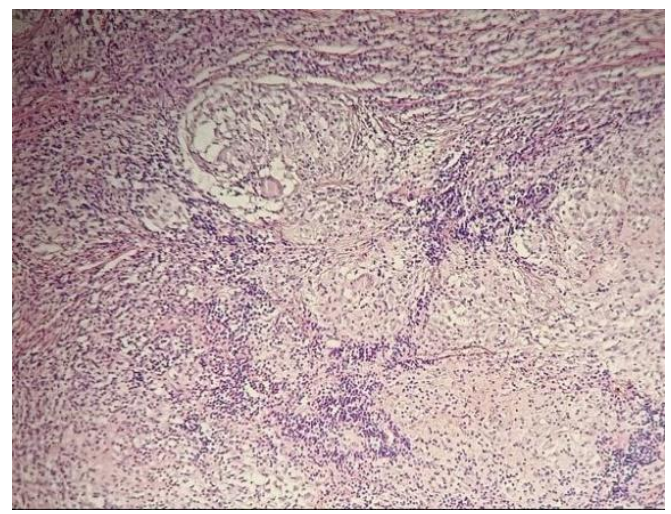

Fig. 2. HE $\times 10$ epithelioid and giganto-cellular granulomatous lesion. The granulomas are confluent in places.

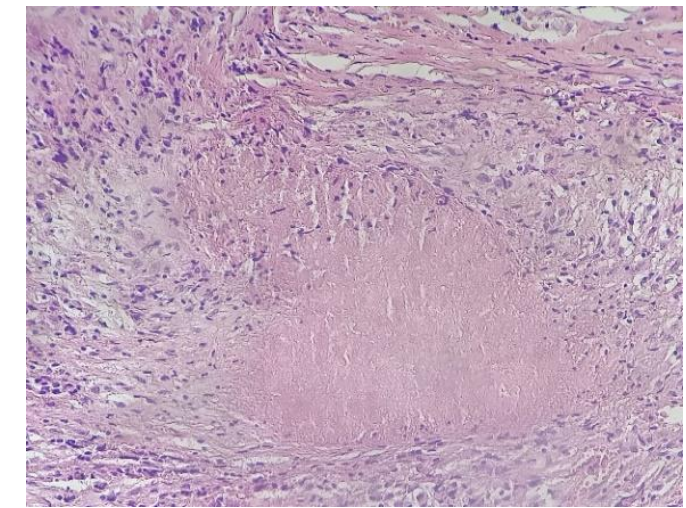

Fig. 3. HE $\times 40$ Caseous necrosis surrounded by epithelioid and giganto-cellular granulomatous.

\section{B. Case File 2}

Patient aged 14 years old, without family history of tuberculosis, admitted to the department for exploration of a right spinal adenopathy, appeared 4 months ago, progressively increasing in size, fistulized a week ago, evolving in a context of unquantified weight loss, accompanied by nasal obstruction and homolateral hearing loss. The clinical examination found a right spinal adenopathy, inflammatory and fistulized skin with pus discharge, painless, and measuring $3 \mathrm{~cm}$ in diameter. The objective otoscopic examination found an otitis media right seromucosa. Nasofibroscopy revealed a tumor bud of the right posterolateral wall of the cavum (Fig. 4). Multiple biopsies of the cavum were performed and the anatomopathology study has shown the presence of granulomas epithelio-giganto-cellular epithelials with caseous necrosis. The results biological showed an inflammatory syndrome manifest.

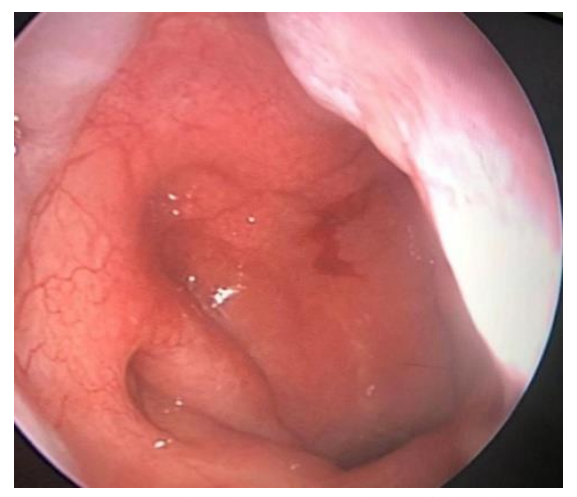

Fig. 4. Endoscopic aspect showinga tumor bud of the right posterolateral wall of the cavum.
Our two patients benefited from a check-up in search of a secondary localization, in particular pulmonary, hence the interest of a chest X-ray and the search for BK sputum, the results were negative in both cases. The diagnosis of Primary nasopharyngeal pseudotumor tuberculosis with nasopharyngeal localization was retained in both patients. Antibacillary therapy was instituted, combining rifampicin, isoniazid and pyrazinamide for two months, followed by a four-month course of rifampicin and of isoniazid. The evolution under treatment was satisfactory, with the control after three months of treatment discontinuation showed a regression of clinical, endoscopic signs.

The control biopsies with histological studies have set up a nasopharyngeal sterilization without histological signs of malignancy. Patients are always followed on a regular basis without no local recidivism with an average retreat of 12 months.

\section{DISCUSSION}

Tuberculosis is a major public health problem in our country. It most often affects the lungs, but other organs are affected in almost one third of cases [3].

Tuberculosis of the nasopharynx corresponds to all the evolving granulomatous lesions of the nasopharyngeal mucosa resulting from the Koch's bacillus infection. It represents a rare localization of the disease in its primary form although the cavum is highly vascularized and located in a very exposed area. It was first described by GRAFF in 1936 [4]. Only a few cases are published in the literature [5].

The mode of nasopharyngeal contamination is due to a local inoculation by inhalation of bacilliferous dust, or a dissemination by blood or lymphatic way from an unknown localization from where the primary localization, or from a known site, mainly pulmonary for the hematogenous way or mucous for the lymphatic way [6], [7].

Clinically, C. Adnane and al published a series from the Department of Ear, Nose and Throat Surgery, Casablanca, Morocco, where the mean age was 22.5 years, with a sex ratio of 2 males to 1 female [6]. For Brahami and al the mean age was 28 years with a male predominance $3 / 4$ [14]. This corresponds with the young age of our series.

Primary tuberculosis of the cavum has a non-specific expression, it usually takes on pseudo-tumoral forms. Tsé and al published in 2003 a series of 17 cases of tuberculosis of the cavum, mainly pseudotumor forms [7]. The clinical signs are similar to those of a nasopharyngeal carcinoma [7], [9], with generally unilateral cervical adenopathy, homolateral nasal obstruction, epistaxis, purulent rhinorrhea with posterior discharge sometimes associated with otological signs such as hypoacusis secondary to seromucous otitis, which is similar to the symptoms found in our two patients.

Endoscopic examination of the cavum can detect one of the lesional aspects of tuberculosis: ulceration, irregular ulcerating swelling, regular mucosal hypertrophy, or even the appearance of common adenoidal vegetations [10]-[12]. In our patients, we found two tumor aspects, a budding tumor of the entire posterolateral wall of the cavum in the first case, and a small budding tumor in the right posterolateral wall of the cavum in the second patient. These 
macroscopic aspects could also correspond to a malignant neoplasia. The radiological signs are not specific and are mostly in favor of a tumoral process, computerized tomography scan and magnetic resonance imaging allow to affirm the absence of invasive character of this tumor, CT and MRI were not requested in our two patients [13].

The positive diagnosis of tuberculosis of the cavum can only be confirmed by histological examination, with the presence of epitheliocellular granulomas with caseous necrosis. Therefore, it is essential to perform multiple biopsies at different sites in order to eliminate a nasopharyngeal carcinoma or a combination of the two, which is possible but exceptional $[6,10]$. The identification of the germ by direct examination or after culture on a specific environment requires 4 to 6 weeks and can be unsuccessful. Advanced techniques such as PCR allow an earlier diagnosis and can sometimes be used to diagnose epithelial-giganto-cellular granuloma without caseous necrosis [5].

The treatment of primary tuberculosis of the cavum is medical, based on multidrug antibacillary therapy. The classic combination used is triple therapy with rifampicin, isoniazid and pyrazinamide for 2 to 3 months, followed by 4 to 6 months of dual therapy (isoniazid and rifampicin) [5], [6]. Therapeutic efficacy is based on the regression of clinical and endoscopic signs; any abnormal evolution should suggest resistance or the possibility of coexisting neoplastic disease, which is why iterative biopsies are necessary [12]. The risk of recurrence is estimated at $1 \%$, mainly due to the appearance of multi-resistant BK strains [4].

\section{CONCLUSION}

Tuberculosis of the cavum is a rare entity but should be known. The firm diagnosis is essentially histological. Treatment is based on anti-bacillary drugs. The prognosis under anti-bacillary treatment is often favorable, failures are mainly related to the emergence of multidrug resistant strains.

\section{REFERENCES}

[1] QUI. Rapport mondial sur la tuberculose. 2014. (Rapport de l'OMS).

[2] Nieves CS, Onofre RDC, Aberin-Roldon FCA, Gutierrez RLC. Nasopharyngeal tubeculosis in a patient presenting with upper airway obstruction. Philipp J Otolaryngol Head Neck Surg 2010; 25(1): 20-22.

[3] Ziad, T., Nouri, H., Adny, A., Rochdi, Y., Aderdour, L., \& Raji, A (2013). Miliaire tuberculeuse aiguë du pharynx ou maladie d'Isambert: à propos d'un cas. Archives de Pédiatrie, 20(1), 41-43.

[4] Bouaity B et coll. Tuberculose du naso-pharynx: à propos d'un cas. Lettre d'ORL et de chirurgie cervico-faciale. 2009; 319 :14-16. PubMed | Google Scholar.

[5] Noël GE. La tuberculose et les infections à mycobactéries. De la microbiologie à la clinique. La Lettre de l'Infectiologue 1990; V: 716-9.

[6] Clément P, Leguyadec T, Zalagh M, Gauthier J, N'Zouba L, Kossowski M. Manifestations bucco-naso-sinusiennes des maladies infectieuses spécifiques. EMC ORL. 2005(2): 198-217. PubMed | Google Scholar.

[7] Tse GM, Ma TK, Chan AB et al. Tuberculosis of the nasopharynx: a rare entity revisited. Laryngoscope. 2003 Apr;113(4):737-40 PubMed | Google Scholar.

[8] C.Adnane,T.Adouly,T.Oubahmane,S.Rouadi,R.Abada,M.Mahtar,M. Roubal. la tuberculose nasopharyngée à propos de 3 cas. Service d'ORL et chirurgie cervicofacial, Casablanca, Maroc. https://doi.org/10.1016/j.aforl.2014.07.278.

[9] Kharoubi S. Miliaire tuberculeuse aigue du pharynx: à propos d?une observation. Rev pneumol Clin. 2004; 60(5):296-298. PubMed | Google Scholar.

[10] Raji A, Essaadi M, Ait Benhamou C, Kadiri F, Laraqui NZ, Benchakroun Y. Aspect rare de la pathologie rhinopharyngée: la tuberculose cavaire. Rev JF ORL 1995; 44: 264-7.

[11] Zanaret M, Dessi P. Tuberculose pharyngée. EMC ORL 1990;20$575 \mathrm{~A} 10,2 \mathrm{p}$.

[12] Marrakchi R, Fathallah M, Ben Ramdane K, Ben Abid H, Sioud H. La tuberculose du cavum, à propos de 2 cas. Rev Laryngol, 1990; 111: $149-52$.

[13] King Ann D, Ahuja Anil T, Tse Gary MK, Van Harselt Adrew CA, Chan Amy BW. MR imaging features of nasopharyngeal tuberculosis: repport of three cases and littérature review. AmJ NeuroRadiol. 2009; 24: 279-282. PubMed | Google Scholar.

[14] I. Brahami, N. Mehtari, F. Mlata, E. Boukli, F. Mébarki, F. Hadj allal, Les complications chirurgicales du cancer de la thyroïde: a propos de 66 cas, Vol. 131, Issue 4, Supplement, October 2014, Page A124. https://doi.org/10.1016/j.aforl.2014.07.261. 